\title{
Characteristics of Response of Piezoelectric Actuators in Electron Flux Excitation
}

\author{
Philip C. Hadinata ${ }^{1} \&$ John A. Main \\ Mechanical Engineering Department, University of Kentucky
}

\begin{abstract}
In this paper the working parameters of non-contact strain control for piezoelectric ceramics are evaluated. The piezoelectric material functions as an actuator that transforms electrical into mechanical energy, and the electrical input is carried out by electron flux on the positive surface. The sample is exposed to some quasi-static inputs, and its responses are recorded using strain gages. The data shows faster and more stable response in the positive regime, but significantly slower response with drift in the negative regime. An electron collector is introduced on the positive surface to enhance the response in the negative regime. Theoretical analyses of energy transfer and electron movements is discussed, and a string of working conditions for controlling the surface strain of piezoelectric material are given as conclusions.
\end{abstract}

Keywords: actuator; control; electron flux; strain.

\section{$1 \quad$ Introduction}

Piezoelectricity is a characteristic of some materials that can convert mechanical into electrical energy, reciprocally. The electrical energy manifests in a differential voltage at the opposite side of the material's surface, and the mechanical energy takes shape as the surface strain. This reciprocal characteristic is a promising feature for lightweight sensor-actuator application such as in space structures, where effects from air drag and gravity are minimal.

As an actuator, piezoelectric material receives electric charge as the input, and transforms it into surface strain. The actuation is conducted using "non-contact" method, in which the input signal is applied to the piezoelectric materials using electron flux instead of conventional cable. The main advantage of using electron flux to stimulate strain is lighter weight and less space. This application is also known to have high spatial resolution and flexible actuation area.

Martin and Main [1], Hadinata and Main [2], Nelson and Main [3] use this method to actuate various types of piezoelectric actuators, but the electricalmechanical energy transformation had never been explored thoroughly, nor the working conditions to utilize this method to the fullest extent. Hadinata and

\footnotetext{
${ }^{1}$ Post-doctoral Research Associate
} 
Main [2], Nelson and Main [3] reported different behavior of the piezoelectric actuator under different surface polarization. While not fatal, these differences can hamper the method's effectiveness to give a desired output.

Several researchers observe the energy and material transfers within piezoelectric material. Ganachaud et.al. [4-6] discuss energy transfers on piezoelectric material within electron-electron collision, electron-phonon (light particle) collision, electron-solid elastic collision, and polarization effects in detail. Ganachaud, Attard and Renoud [5] present a model of space charge build-up in an insulating target under electron bombardment. The charging of the plate is modeled using Monte Carlo simulation. Attard and Ganachaud [6] state that as the target charge builds up, the potential at the surface and the secondary yield vary. All of these phenomena will lead to electron excitation, and eventually become known as "secondary electron emission".

The electron flux also polarizes the material. The homogeneous electric field strength between positive and negative surface is given by:

$$
\overline{\mathrm{E}}=\frac{\mathrm{V}_{\mathrm{b}}-\mathrm{V}_{\mathrm{p}}}{\mathrm{h}}
$$

where $\overline{\mathrm{E}}=$ electric field across the plate, $\mathrm{V}_{\mathrm{p}}=$ potential on positive surface, $\mathrm{V}_{\mathrm{b}}=$ the potential on negative surface (backpressure voltage) $\mathrm{h}=$ piezoelectric thickness. Main, Nelson and Martin [7-8], Nelson and Main [9] demonstrate that strains in piezoelectric materials could be controlled through a combination of applied electron fluxes and potentials. It was also shown that the changes in structure remained after the input signals were removed, indicating that there is some potential for energy efficient static strain control in adaptive structures using this method.

The physics of strain development in charged piezoelectric material is described in detail in several papers. Batra et al. [10] present the optimum location of a given piezoceramic actuator that will require the minimum voltage to null the deflections of a simply supported rectangular linear elastic plate vibrating near one of its fundamental frequencies. Ghosh and Batra [11] emphasize on shape control of piezoceramic plates. A fiber-reinforced laminated composite plate with 4 small piezoceramic actuators attached on top surface is used for experimental sample. Crawley and deLuis [12] stage a model of static and dynamic behavior of segmented piezoelectric actuator under load influences, either bonded to an elastic structure, or embedded in a laminated composite. Crawley and Lazarus [13] elucidate the induced strain on isotropic and anisotropic plates subjected to different loads using Rayleigh-Ritz method. Lee 
and Moon [14] show that distributed piezoelectric sensors/actuators could be adopted to measure specific modes of one-dimensional plate or beam.

The objective of this paper is to evaluate the characteristics of the non-contact piezoelectric actuation method, so a proper input signal and condition can be implemented to obtain desired responses. The electron flow in-and-out of the actuator is studied by applying quasi-static backpressure voltage. Time response of the material is evaluated by changing the backpressure voltage in an abrupt manner, and strain distribution is investigated by exposing the material to a short burst of electron flux, and let the charge spread on the surface.

\section{Current Response of the Piezoelectric Material}

The test specimen is a rectangular PZT-5H plate, $75 \mathrm{~mm}$ long, $50 \mathrm{~mm}$ wide, and $1.975 \mathrm{~mm}$ thick. The plate was obtained from the manufacturer with silver electrodes distributed on both sides. The positive electrode was removed with a combination of swabbing with nitric acid and light sanding to expose the dielectric material to the electron beam. The remaining electrode was connected to a pico-ampere meter and a power amplifier to allow the potential of the electrode to be controlled and the amplifier-electrode current to be observed. A sketch of the experimental setup is included as Figure 1.

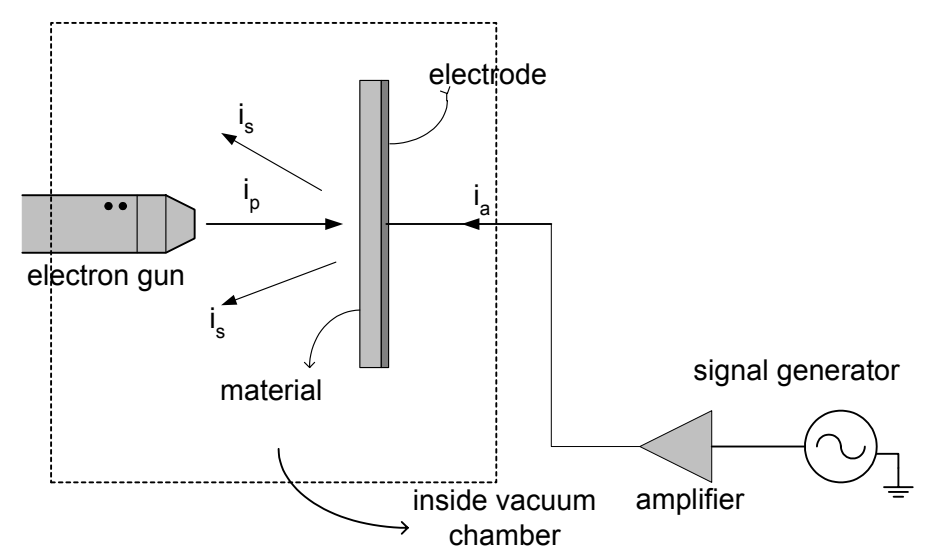

Figure 1 Experiment setup.

In Figure $1 i_{p}$ is the primary electron current (inbound electrons coming from the electron gun), $i_{s}$ is the secondary electron current (exiting electrons from the material) and $i_{a}$ is the electron current through the electrode lead. Note that electron current differs from conventional electric current. As a convention, positive $i_{p}$ flows from the electron gun toward the material. Charge conservation demands that the three currents are related by 


$$
\mathrm{i}_{\mathrm{a}}=\mathrm{i}_{\mathrm{s}}-\mathrm{i}_{\mathrm{p}}
$$

when the system is at equilibrium. The electron gun is used to control the potential at a given point on the bare ceramic surface, or positive surface $\left(\mathrm{V}_{\mathrm{s}}\right)$. The power amplifier controls the potential on the negative surface, or backpressure voltage $\left(\mathrm{V}_{\mathrm{b}}\right)$.

In this experiment the strain and current responses of the piezoelectric plate subjected to an electron flux were examined under a range of conditions. The strains were recorded at 16 locations using 350 - $\mu$ strain gages with gage factor 2.095, arranged in 4x4 matrix, shown in Figure 2. A 24-channel strain gage data acquisition system was used to record all of the strain signals simultaneously when the various inputs were applied to the plate. The electron gun emission current was set to flood (covered the entire area with uniform intensity) and

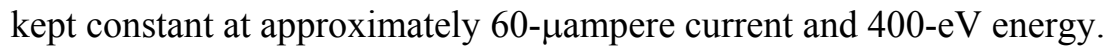

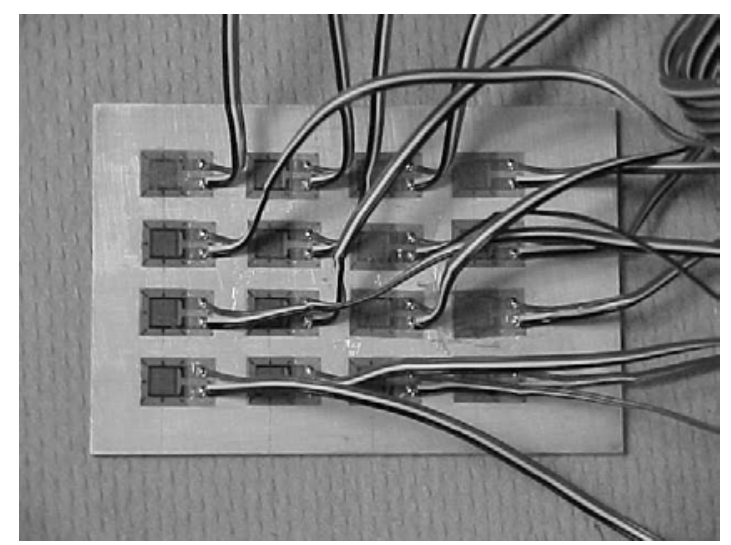

Figure 2 Piezoelectric ceramic with 16 strain gages on the negative surface.

The positive output of the power amplifier was connected to the negative ground of pico-ampere meter. This means that a positive sign on the current traces indicates flow of conventional current from the electrode to the power amplifier, or positive $i_{p}$ as shown in Figure 1. This setup was exposed to a vacuum condition in a vacuum chamber, $3 \times 10^{-7}$ torr $(\mathrm{mm} \mathrm{Hg})$. For initialization, the electron gun was fired when the backpressure voltage $\left(\mathrm{V}_{\mathrm{b}}\right)$ was set to ground. This sequence caused electrons to reside on the material until equilibrium was achieved, thus putting a small amount of strain on the surface (about 15-20 $\mu$ strain). This strain level was considered to be the zero strain for all subsequent measurements. 
Three sets of data are presented in the Figures 3 - 5. Only a single strain trace is shown in each figure since the electron beam flooded the entire bare face of the piezoelectric and only single electrode covers the negative face, so the condition is assumed as uniform on all locations on the plate. The strain traces were measured in-plane. $\mathrm{V}_{\mathrm{b}}$ was varied slowly using a sine wave with $20 \mathrm{mHz}$ frequency and 200 volt peak-to-peak with various DC offsets: zero on Figure 3, $100 \mathrm{~V}$ on Figure 4, and $-100 \mathrm{~V}$ on Figure 5. It is shown that, for all cases, the strain output is very stable and dependent upon $V_{b}$ when the backpressure potential $\left(\mathrm{V}_{\mathrm{b}}\right)$ was predominantly positive, Figures 3 and 4 . In the tests with predominantly negative $\mathrm{V}_{\mathrm{b}}$, the strain still responds as a function of $\mathrm{V}_{\mathrm{b}}$, but significant drift is evident, Figure 5.

The current results also show a sharp contrast between actuation with positive and negative $\mathrm{V}_{\mathrm{b}}$. In all of the tests the current remained at extremely low levels (approximately 0.1 mampere or less) when $\mathrm{V}_{\mathrm{b}}$ was below $40 \mathrm{~V}$. As $\mathrm{V}_{\mathrm{b}}$ transitions to greater than $40 \mathrm{~V}$ the current flow through the material suddenly decreases to approximately $-12 \mu$ ampere, as can be seen in Figures 3 and 4 . Further increases above 40 volts lead to a slight gradual decrease in the current until approximately -18 uampere as can be seen in Figure 4.
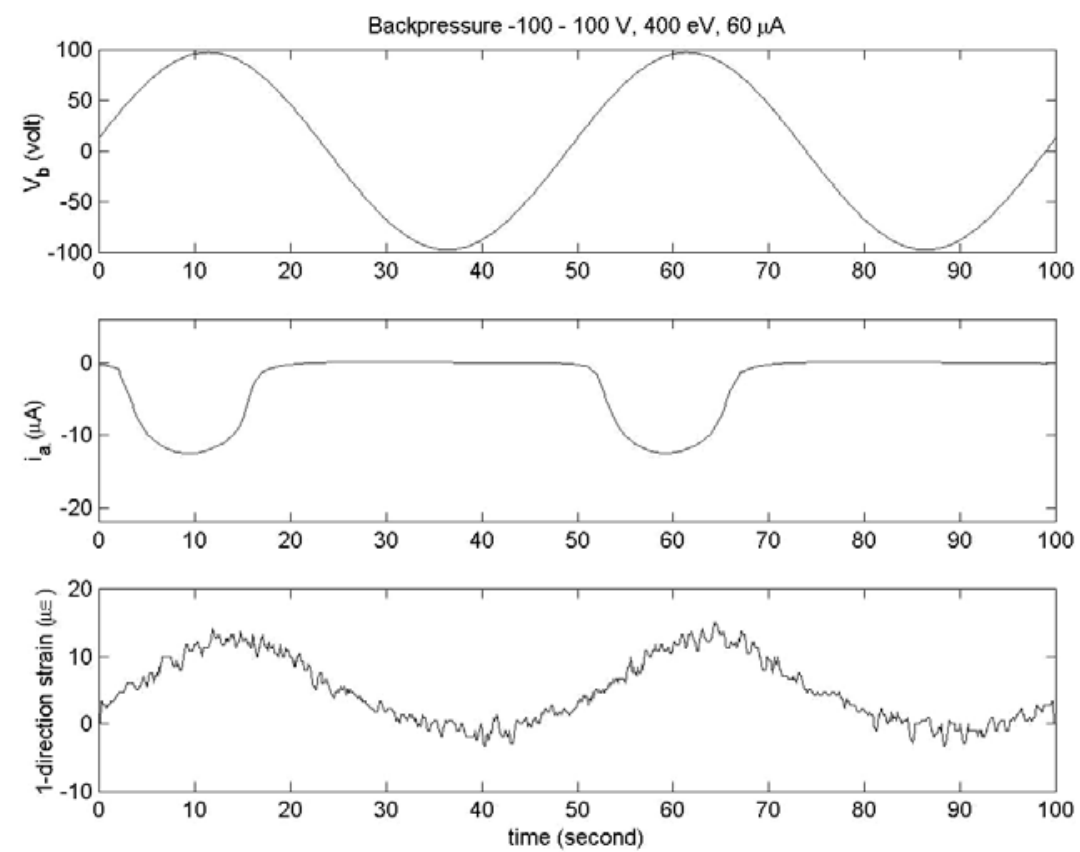

Figure 3 Time histories of strain and current due to $200 \mathrm{Vp}-\mathrm{p}, 0 \mathrm{DCV}$ offset $\mathrm{V}_{\mathrm{b}}$. 

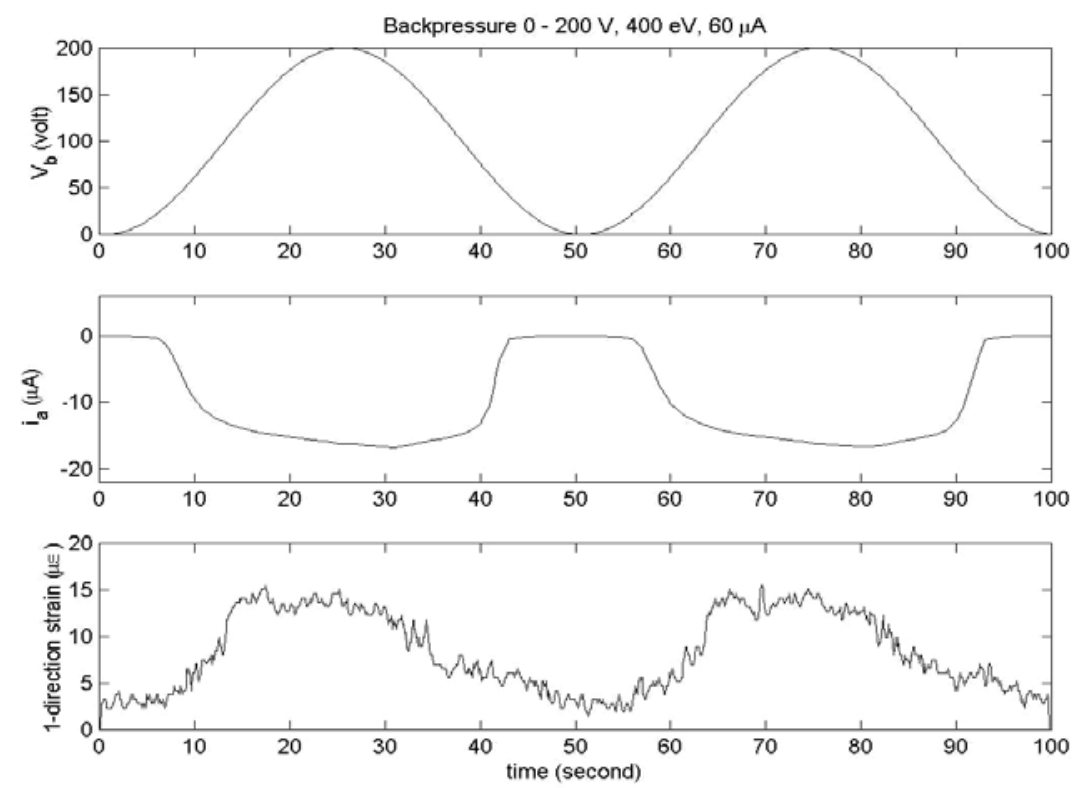

Figure 4 Time histories of strain and current due to $200 \mathrm{~V}$ p-p, $100 \mathrm{DCV}$ offset $\mathrm{V}_{\mathrm{b}}$.
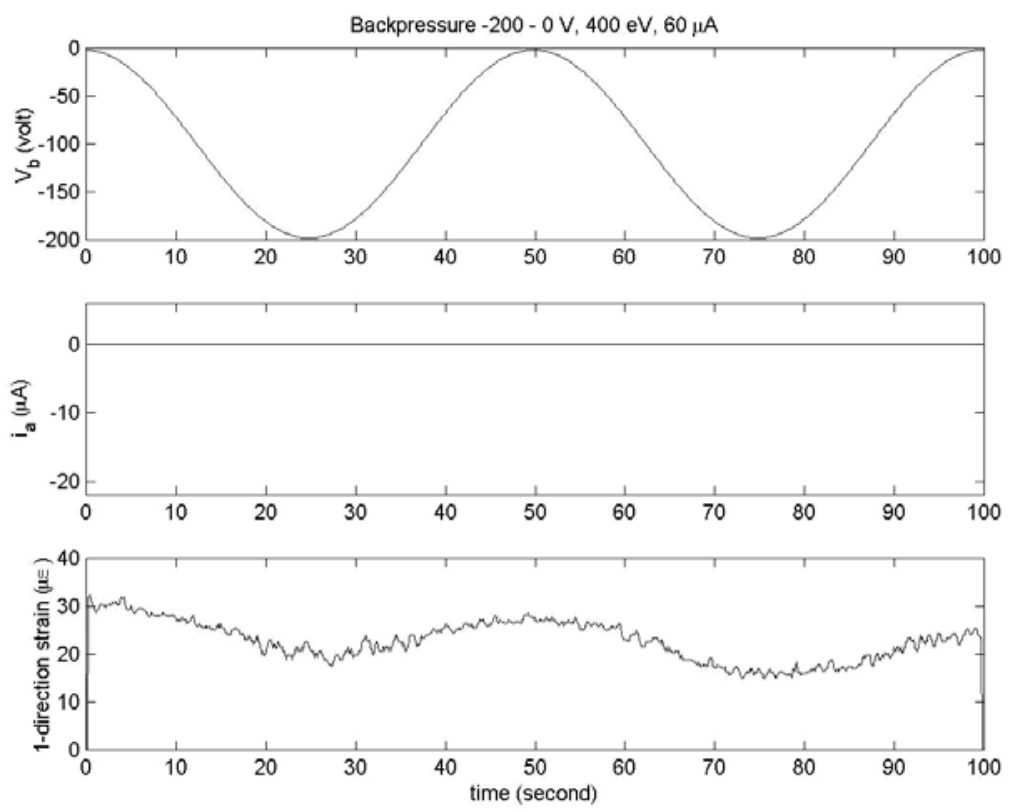

Figure 5 Time histories of strain and current due to $200 \mathrm{~V} p-p,-100 \mathrm{DCV}$ offset $\mathrm{V}_{\mathrm{b}}$. 


\section{Time Response of Piezoelectric Material}

For time response experiment, the same experiment setup was used, only the backpressure voltage $\left(\mathrm{V}_{\mathrm{b}}\right)$ was varied to some degree, as elucidated in Figure 6.a. After 20 seconds of ground level, it was stepped up abruptly to $100 \mathrm{~V}$. This level lasted for 20 second, then it went back to ground. After 20 second, it was stepped down to $-100 \mathrm{~V}$. This lasted also for 20 seconds, then it was restored to ground level. There appears to be two distinct time responses related to the polarity of $\mathrm{V}_{\mathrm{b}}$. If $\mathrm{V}_{\mathrm{b}}$ was stepped up (from 0 to $100 \mathrm{~V}$ ) the strain response quickly mimics the $V_{b}$ pattern, Figure 6.b. But if $V_{b}$ was stepped down, the strain needed a considerably longer time to reach steady state value. Hadinata and Main [15] reported that the material needed only 1 second to adjust the strain in the step-up experiment, but almost 60 seconds in the step-down experiment.
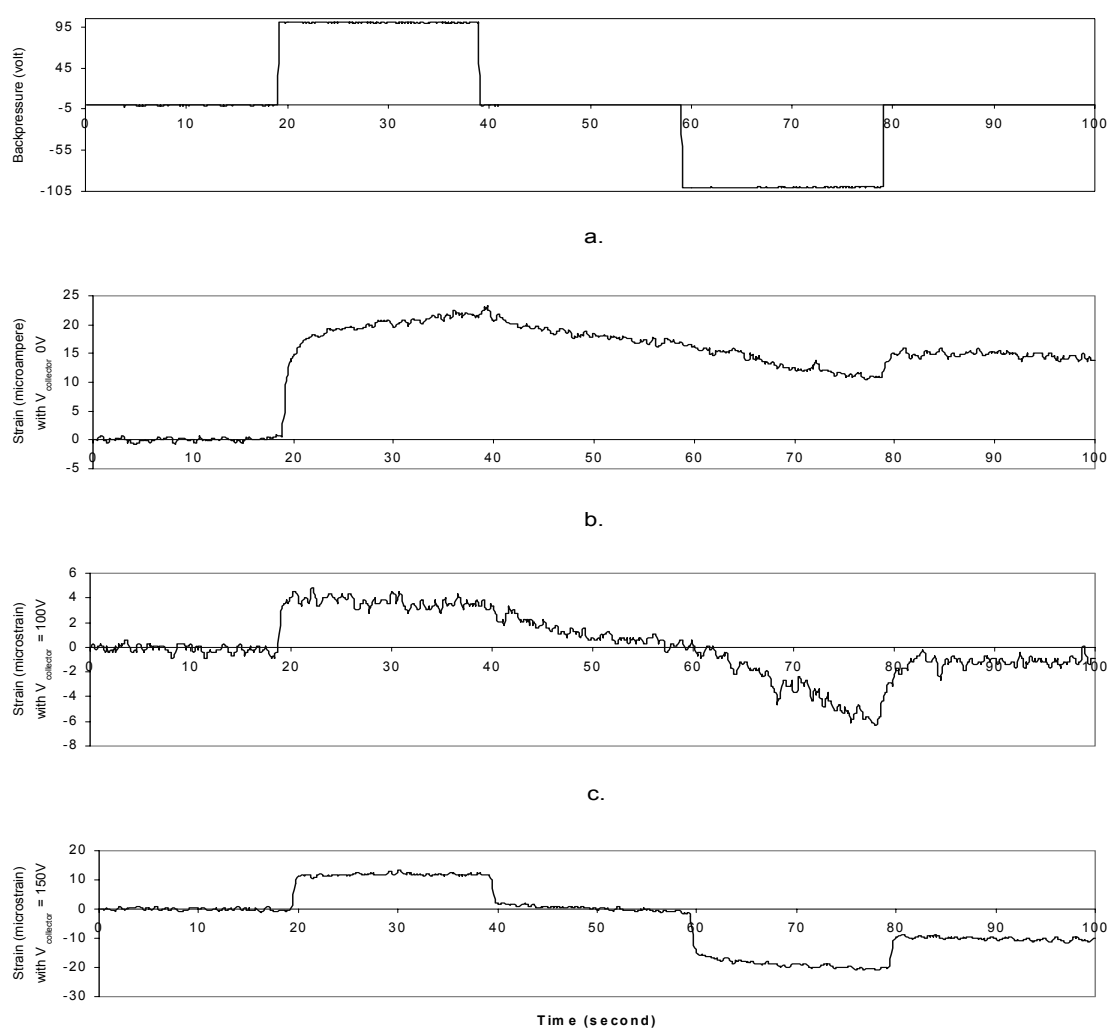

d.

Figure 6.a Backpressure voltage;

6.b Strain response with no collector potential;

6.c Strain response with $100 \mathrm{~V}$ collector potential;

6.d Strain response with $150 \mathrm{~V}$ collector potential. 
A simple electron collector was placed between the electron gun and the specimen, $2 \mathrm{~cm}$ from the specimen, Figure 7. The collector was connected to a positive source to force the primary electron to hit the material with greater speed, and to attract the secondary electron emission from the specimen, thus making the electron flow better. A significant increase in strain response was detected as seen in Figures 6.c and 6.d. When the collector potential was increased to $100 \mathrm{~V}$, the strain responded to change in $\mathrm{V}_{\mathrm{b}}$ with faster rate, Figure 6.c., although it is not fast enough to follow $V_{b}$ pattern. Finally, with collector potential $150 \mathrm{~V}$, the strain pattern strongly resembled that of the $\mathrm{V}_{\mathrm{b}}$ pattern, Figure 6.d.

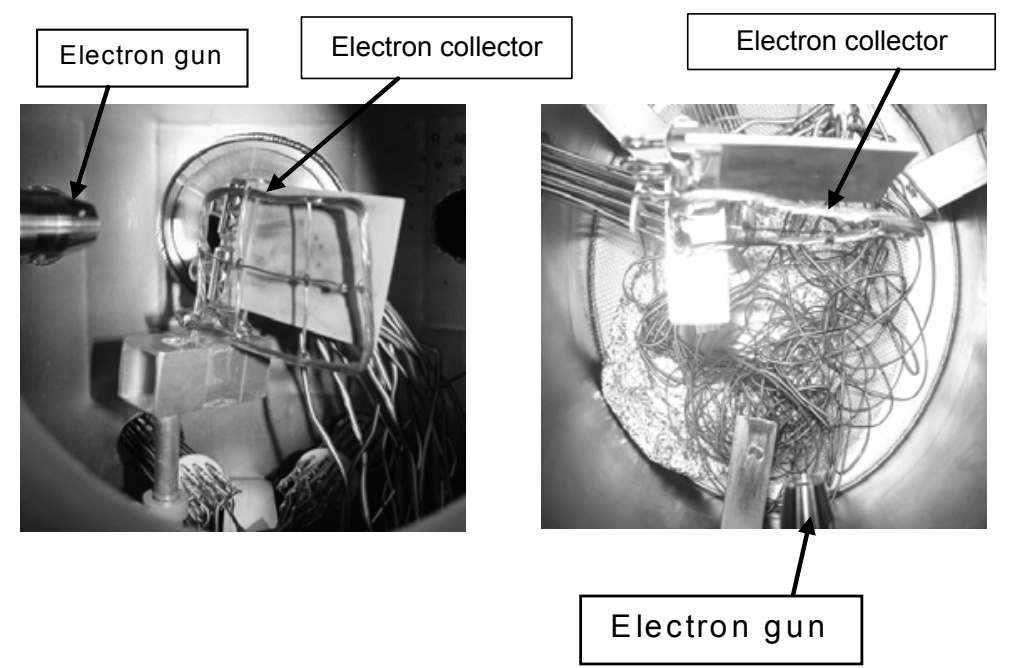

Figure 7 Electron collector emplacement in vacuum chamber.

\section{$4 \quad$ Strain Distribution}

The following experiment was conducted to investigate strain growth on the surface in more detail. First, the specimen was subjected to backpressure $V_{b}=0$ when illuminated by a flooded electron beam. This set the zero point for the test. Then the electron gun was turned off and $V_{b}$ was increased to 200 volt. The next step was to shoot the plate in the center with 400-eV energy, 60ampere emission current, with approximately $1 \mathrm{~cm}$ in diameter for 2 seconds. The strain distribution sequence is presented in Figure 8. At first only the area subjected to the electron flux responds, denoted by high strain on the center. Then the strain distributes along the plate until the whole surface has high strain. After 1 seconds, the strain distribution reached equilibrium, as is depicted in Figure 8.c. 


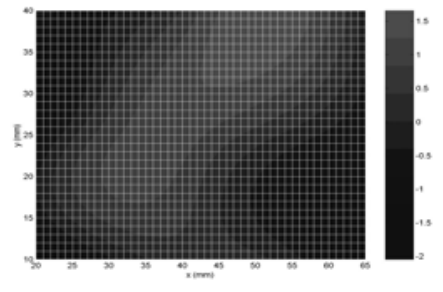

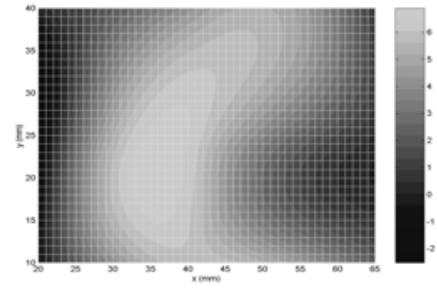

b.

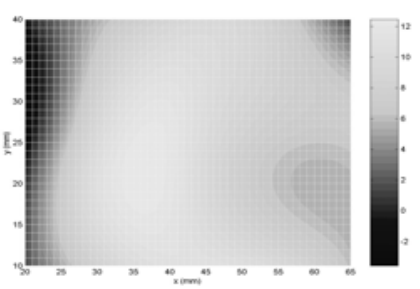

c.

Figure 8 Strain distribution sequence with electron beam in the center: a. $\mathrm{t}=0 \mathrm{~s}$, b. $\mathrm{t}=0.5 \mathrm{~s}$, c. $\mathrm{t}=1 \mathrm{~s}$.

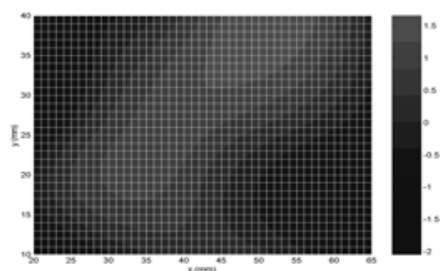

a.

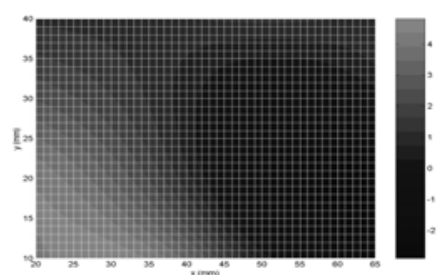

b.

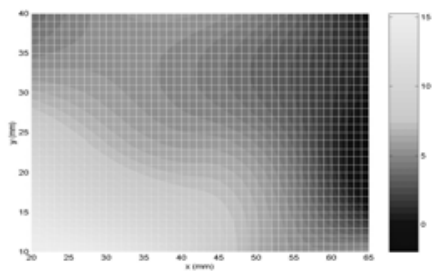

Figure 9 Strain distribution sequence with electron beam on the corner:
a. $\mathrm{t}=0 \mathrm{~s}$,
b. $\mathrm{t}=0.5 \mathrm{~s}$,
c. $\mathrm{t}=1 \mathrm{~s}$.

A clearer view of how the charge (and strain) distributes along the material is provided by placing the electron flux on the corner of the material. The results are presented in Figure 9. Again, it is clearly seen that the area under illumination (i.e. strain gage number 1 or the left lower corner of the plate) 
developed the strain first. Then the rest of the area follows. This phenomenon is called 'blooming'. Note that the expansion of the strain as a function of time is similar to the surface charge blooming predicted by Attard and Ganachaud [6].

\section{Discussion}

The mechanics of electron current at the vacuum-material boundary can be represented using quantum physics. Using Schrödinger postulate [16], the electron current in vacuum can be represented as:

$$
\begin{aligned}
& \Psi_{1}=Y_{1} e^{i \alpha x}+Y_{2} e^{-i \alpha x} \\
& \text { where } \alpha=\sqrt{\frac{2 m E_{p}}{\hbar^{2}}}
\end{aligned}
$$

When the electron strikes the piezoelectric it is exposed to the potential barrier $\mathrm{U}$ inside the material. Therefore:

$$
\begin{aligned}
& \Psi_{2}=Z_{1} e^{i \beta x}+Z_{2} e^{-i \beta x} \\
& \text { where } \beta=\sqrt{\frac{2 m\left(E_{p}-U\right)}{\hbar^{2}}}
\end{aligned}
$$

Equation (3) and (5) are visualized in Figure 10.b.

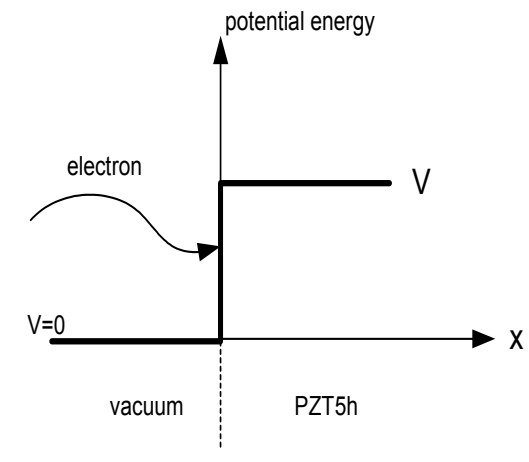

a.

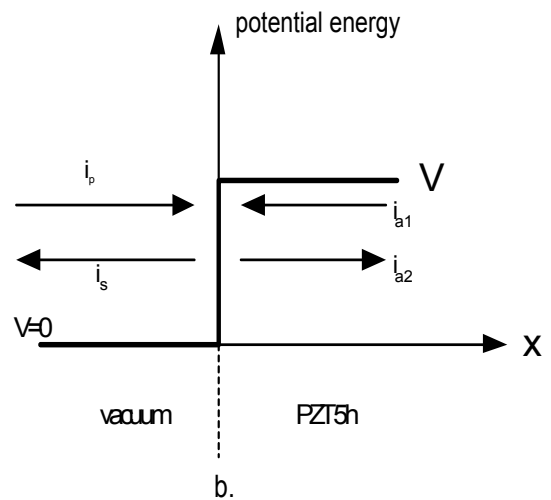

b.

Figure 10.a Energy representation of an electron impacting the PZT plate

10.b Electron current orientations at the energy barrier. 
The first term in Equation (3) describes the primary electron, $i_{p}$, while the second term describes the secondary electron current, $i_{s}$. The first factor of Equation (5) is the electron current from PZT to amplifier $\left(i_{1}\right)$, and the second factor denotes the electron current in the opposite direction $\left(\mathrm{i}_{\mathrm{a} 2}\right)$. Thus, the electron current $i_{a}$ is the combination of these two factors:

$$
i_{a}=i_{a 1}+i_{a}
$$

The summation of all three components of electron current leads up to Equation (2).

The nature of electron current on Figures 3-5 can be explained using energy expressions. The kinetic energy of electron can be represented as a function of surface potential:

$$
\mathrm{K}=\frac{1}{2} \mathrm{mv}^{2}=\mathrm{e} \mathrm{V}_{\mathrm{p}}
$$

where $\mathrm{e}=$ electron charge and $\mathrm{V}_{\mathrm{p}}=$ the potential at the surface of the ceramic surface (front surface, exposed to electron beam). So the kinetic energy of electron varies linearly with the potential of the bare surface of the plate. The PZT can be considered as a capacitor with potential energy:

$$
\mathrm{U}=\frac{1}{2} \mathrm{C}\left(\mathrm{V}_{\mathrm{p}}-\mathrm{V}_{\mathrm{b}}\right)^{2}
$$

$\mathrm{C}=$ material capacitance, $\mathrm{V}_{\mathrm{b}}=$ backpressure potential, $\mathrm{V}_{\mathrm{p}}=$ positive-side potential.

So the potential energy of PZT varies quadratically with the potentials on the front and back of the plate.

The energy balance is shown conceptually in Figure 11 . If $\mathrm{V}_{\mathrm{b}}$ is initially set to zero, then the potential energy of the plate as a function of the plate positive surface potential $\left(V_{p}\right)$ is a parabola with the vertex at the origin (Curve $U$ ). The kinetic energy of the incoming electron (Equation 8) is represented by a straight line. If an electron flux with initial energy in the positive yield range strikes the plate then the surface will become increasingly positive until a balance is achieved between the kinetic energy of the incoming electron and the potential energy of the plate. This system state is therefore at point $\mathrm{A}$ and the plate surface potential is given by the location of point $\mathrm{A}$ on the horizontal axis. The driving force behind the current is the electric field in the material, $\left(\mathrm{V}_{\mathrm{p}}-\mathrm{V}_{\mathrm{b}}\right) / \mathrm{h}$.

Increasing $\mathrm{V}_{\mathrm{b}}$ moves the potential energy curve to the right, represented by $\mathrm{U}_{\mathrm{II}}$, and the stable state moves from point A to A'. A new equilibrium state is 
achievable under these circumstances. Increasing $\mathrm{V}_{\mathrm{b}}$ will reduce the secondary electron emission yield. More primary electrons stick to the plate, so the excess electrons will flow towards the power amplifier. The negative readings on the pico-ampere meter in the positive $\mathrm{V}_{\mathrm{b}}$ region support this phenomenon, Figures 3 and 4 . The very stable $\mathrm{V}_{\mathrm{b}}$-strain behavior experienced at $\mathrm{V}_{\mathrm{b}}$ values above 40 volts supports the conclusion that the system is in a very stable regime in this $V_{b}$ range and the increase in the electric field in the material supports the increase in the leakage current. Reducing $\mathrm{V}_{\mathrm{b}}$ means making the plate surface more negative, so the next incoming electron comes with slower speed. The potential energy curve moves to the left, represented by $\mathrm{U}_{\mathrm{III}}$, and eventually no balance between the incoming kinetic energy and the plate potential energy is possible. This lack of a stable equilibrium is demonstrated by the drift in the strain output seen in Figure 5.

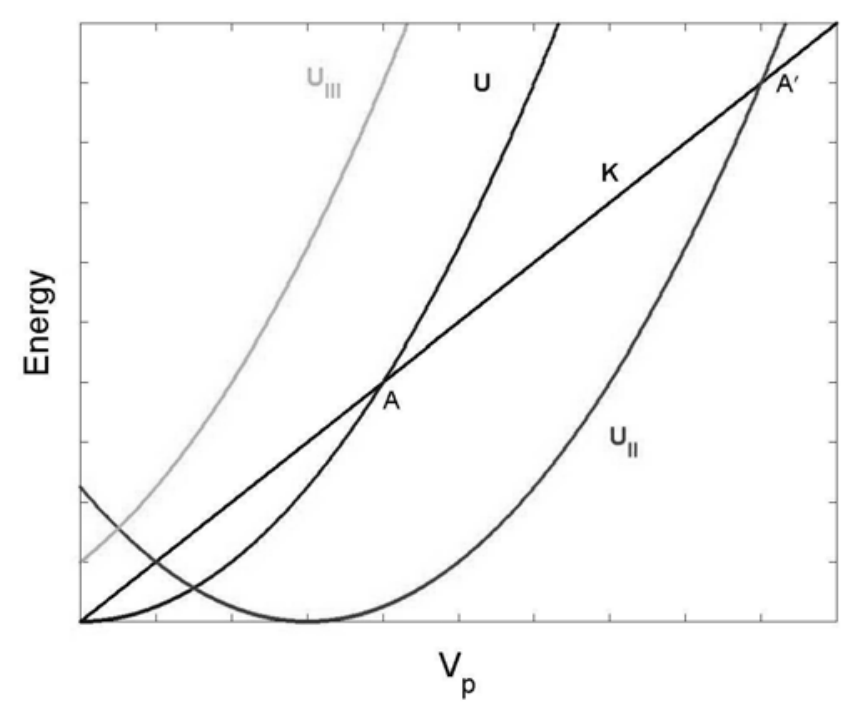

Figure 11 Electron kinetic energy and PZT potential energy chart.

To explain the time response behavior, a secondary electron chart from Bruining [17] is presented in Figure 12. The constant electron beam can be considered as an excitation to the plate. Its presence quickly changes the energy equilibrium of the whole system, until it resides on $\mathrm{E}_{\mathrm{II}}$ point on Figure 12. When $\mathrm{V}_{\mathrm{b}}$ is increased, the plate becomes more positive. The primary electron energy will increase due to the faster velocity of the electrons. The secondary electron yield will fall below 1 . This means the primary electrons are absorbed, and their energy is not enough to eject electrons residing on the plate. More 
primary electrons stick to the plate, so the excess electrons will flow towards the power amplifier.

On the other hand, when the backpressure voltage is reduced, primary electrons of an energy less than $\mathrm{E}_{\mathrm{II}}$ strike the plate, and their energy is transferred to eject slightly more electrons than that of the incident. This energy exchange and electron excitation process takes time, so the result is a slower strain change when the electrode is stepped down, which decelerates the incident electrons, than when it is stepped up. These processes manifest themselves in the secondary yield curve, where electrons with energies greater than $E_{\text {II }}$ generate negative charges on a dielectric surface, and those with energies less than $E_{I I}$ stimulate positive surface charges.

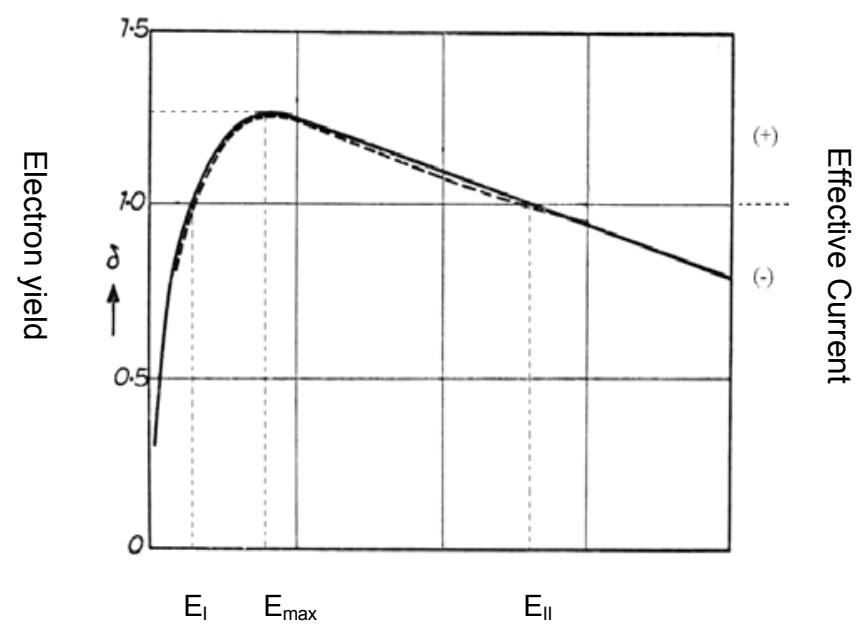

Figure 12 Plot of secondary electron yield against incoming energy by Bruining ${ }^{[17]}$.

The positive electron collector simply attracts the secondary electrons, making the energy transfer between primary and local electron faster. The addition of positive potential in front of the PZT surface speeds up the incoming electron, thus forcing the secondary electron to be less than 1 at all times (i.e. incoming electron energy is always greater than $\mathrm{E}_{\mathrm{II}}$ ). Furthermore, the positive electron collector adds potential electric energy to the local electrons, helping them easily overcome the energy barrier to transform into free electron (as secondary electron). At $100 \mathrm{~V}$, the strain response has not exactly mimic the pattern of the change in backpressure voltage, which is expected because the electron collector does not provide enough potential energy, both to the inbound primary electrons and to the local electron to overcome the energy barrier. At $150 \mathrm{~V}$ and more, the electron collector induces enough energy for the primary electrons to 
speed up and for the secondary electron to excite, thus allowing the strain response to follow the backpressure pattern.

\section{Conclusion}

A set of experiment is conducted to exploit the characteristic of the non-contact method of piezoelectric actuation using electron flux. Based on the three sets of experiments, it is concluded that the PZT-5h works best in the positive backpressure regime (fast strain and current response). Its response in the negative regime shows significant lag, proved by slow time response and negligible current flowing out of the material. It is shown that kinetic energy of primary electron plays the key role in this research. If actuation in the negative regime is desired, an electron collector is compulsory for the system. The function of this electron collector is twofold: to attract secondary electrons and to speed up the primary electrons. The electron flow is thus improved, and similar strain and current response with those on the positive regime is achieved.

The highest magnitude in strain responses is achieved at the location of the electron flux, but after some time, most of the surface has the same strain magnitude with that directly under electron flux influence. This is paramount if a uniform strain distribution is not desirable. High spatial resolution can be achieved using a short expose of electron flux (less than 1 second, depending on the desired resolution).

\section{Acknowledgments}

The authors wish to thank National Science Foundation, especially the program chair Dr. Allison Flatau, for the fund and opportunity they spent throughout this research

\section{References}

1. Martin, Jeffrey W. \& Main, John A., Noncontact Electron Gun Actuation of a Pieazoelectric Polymer Thin Film Bimorph Structure, Journal of Intelligent Material Systems and Structures, 13(6), pp. 329-338 (June 2002).

2. Hadinata, Philip C. \& Main, John A., Strain and Current Responses During Electron Flux Excitation of Piezoelectric Ceramics, Journal of Intelligent Material Systems and Structures, 13(6), pp. 339-348 (June 2002).

3. Nelson, George A. \& Main, John A., Non-contact Electron Gun Strain Control of Piezoceramics, AIAA Journal, 39(9), pp. 1808-1813 (2001). 
4. Ganachaud, J. P. \& Mokrani, A., Theoretical Study of the Secondary Electron Emission of Insulating Targets, Surface Science, 334(1-3), pp. 329-341 (July 10, 1995).

5. Ganachaud, J. P., Attard, C. \& Renoud, R., Study of the Space Charge Induced by an Electron Beam in an Insulating Target part I: Monte Carlo Simulation, Phys. Stat. Sol (b), 199, pp 175 - 184 (1997).

6. Attard, C., Ganachaud, J. P., Study of the Space Charge Induced by an Electron Beam in an Insulating Target part II: Presentation of the Results, Phys. Stat. Sol (b), 199, pp 455 - 465 (1997).

7. Main, J. A., Nelson, G. \& Martin, J., Maintenance of Inflated Structure Shape Using Electron Gun Controlled Piezoelectric Materials, Proceedings of the $39^{\text {th }}$ AIAA Structures, Structural Dynamics, and Materials Conference, Adaptive Structures Forum (April 20-23 1998), Long Beach, CA, AIAA Paper 98-1983.

8. Main, J. A., Martin, J. W. \& Nelson, G. C., Noncontact Shape Control of Membrane Mirrors, Ultra Lightweight Space Optics Challenge Workshop, Napa, California (March 24-25 1999).

9. Nelson, G. C., Main, J. A., Noncontact Electron Gun Strain Control of Piezoceramics, AIAA Journal, 39(9), pp. 1808-1813 (2001).

10. Batra, R. C., Liang, X. Q. \& Yang, J. S., Shape Control of Vibrating Simply Supported Rectangular Plates, AIAA Journal, 34(1) (January 1996), pp. 116-122.

11. Ghosh, K. \& Batra, R. C., Shape Control of Plates using Piezoceramic Elements, Proceedings of SPIE - The International Society for Optical Engineering Active Materials and Smart Structures, 2427 (1995), pp. 107-121.

12. Crawley, E. F. \& deLuis, Javier, Use of Piezoelectric Actuators as Elements of Intelligent Structures, AIAA Journal, 25(10) (October 1987).

13. Crawley, E. F. \& Lazarus, K. B., Induced Strain Actuation of Isotropic and Anisotropic Plates", AIAA Journal, 29(6) (June 1991), pp.944-951.

14. Lee, C. K. \& Moon, F. C., Modal Sensors/Actuators, Journal of Applied Mechanics, 57 (June 1990), pp.434-441.

15. Hadinata, Philip C. \& Main, John A., Time Response of Electron Gun Strain Control of Piezoelectric Materials, Proceedings of SPIE Smart Structures and Integrated System, Smart Structures and Materials, 3985 (2000), pp. $378-384$.

16. Eisbert, Robert \& Resnick, Robert, Quantum Physics of Atoms, Molecules, Solids, Nuclei, and Particles, John Wiley \& Sons Inc. (1974).

17. Bruining, Hajo, Physics and Application of Secondary Electron Emission, McGraw-Hill Book Co., New York (1954). 